gak aspirć en R d'arriver au charbon $\mathrm{K}$, el un noyau $\Lambda$ constilué par un faisceau de fils de fer et logé dans le haut du lube. On remarque autour de $\mathrm{R}$ Jes spires d'un solénoïde $\mathrm{S}$ traversé constamment par le courant primaire alternatif, pris aux bornes $\mathrm{K}_{1} \mathrm{~K}_{2}$.

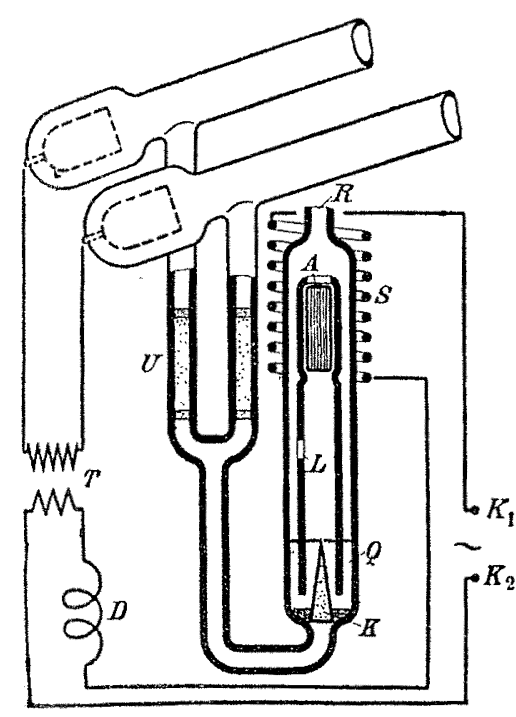

Fig. 1.

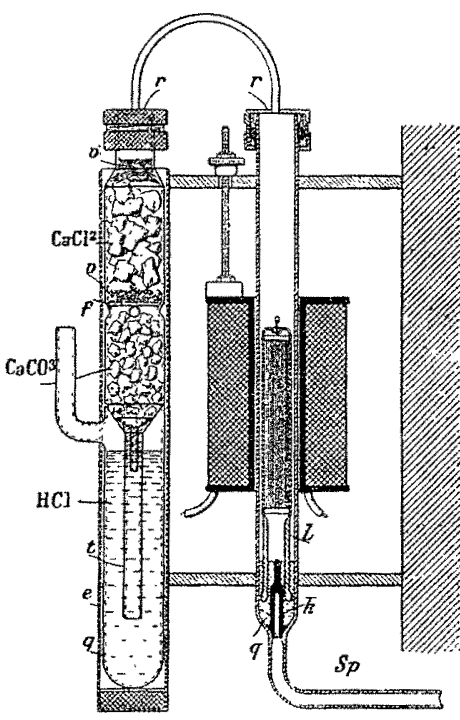

Fig. 2.
$\mathrm{Si}$, par suite du fonctionnement, la raréfaction est pousscé trop loin, la conductivilé du tube Moore se rapproche de son point critique, et devient trop grande ; le courant augmente dans le tube et dans le solénoïde, de sorte que le noyau A se trouve soulevé ; son ascension fait baisser le niveau du mercure $Q$, qui découvre plus ou moins la pointe dn charbon K. Quand la pression est remontée, le courant diminue dans le solénoïde qui laisse retomber son noyau ; te mercure recouvre de nouveau le charbon, et l'accès du gaz est arrêté. Le tube respire ainsi au moins deux fois par minute.

Le gaz admis par la valve se partage entre les deux branches du tube $U$, soudé très près des électrodes aux extrémités du lube éclairant. Pour empêcher les décharges par ce trajet, on crée une résistance en garnissant les deux jambes du lube en U de sable maintenu par deux bouchons.

Le courant primaire emprunté à un réseau alternalif $\mathrm{K}_{1} \mathrm{~K}_{2}$, traverse le solénoüde $\mathrm{S}$, la bobine de self-induction $\mathrm{D}$ et le primaire du transformateur. I.e tube est directement soudé anx bornes secondaires. La tension est élevée de tro à I? 000 volls environ.

l'azote s'obtient facilement en faisant passer l'air almosphérique sur un bâlon de phosphore qui absorbe l'oxygène. Quant a l'anhydride carbonique, il se própare au moyen du disposilif de la figure 2, emprunté à l'Electrical Engineering du 26 mai г9ro.

On emploie un flacon générateur de $23 \mathrm{~cm}$ de hauteur et de $3 \mathrm{~cm}$. de diamètre. Sur la figure ?, les leltres représentent : $e$, enveloppe en cuivre; $f$, feulre; $l$, carbone; $l$, tube de verre; $q$, mercure ; $r$, raccord en cuivre; $s p$, soupape ; $v$, laine de verre. Au moment oì il y a aspiration, la pression almosphérique fait monter l'acide chlorhydrique (IICCI) an contact du carbonate de chaux $\left(\mathrm{CO}^{3} \mathrm{Ca}\right)$, el il se dégage l'anhydride carbonique $\mathrm{CO}^{2}$.

Le professeur Wedding a effectuć un grand nombre d'expériences dans une des salles du laboratoire électrolechnique de la Konigliche Technische Hochschule, de Charłottembourg, pour le compte de l'Allgemeine Moore Lichl Gesellschafl. Il a obtenu le résultat suivant : une tranche de $\mathrm{x} \mathrm{cm}$ de lirgeur a une intensité de $0,5 \mathrm{r} / 4$ bongie internationale et le mètre courant 51,4 bougies, le circuil d'alimentation ayant les constantes ci-dessous :

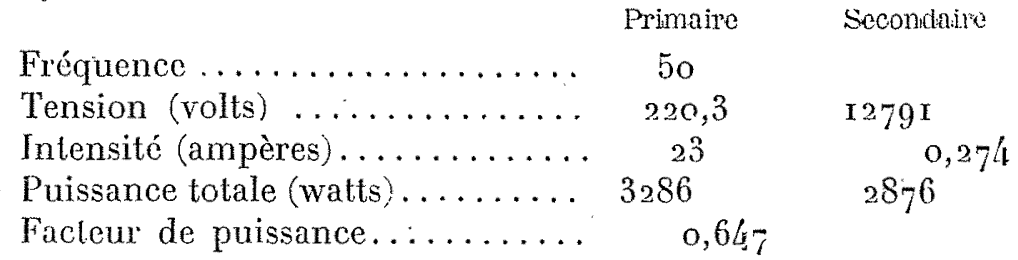

La longueur totale du tube était de $37^{\mathrm{m}} 50$; la puissance primaire absorbée, 3286 watts, soit 87,6 watls par mètre courant de $5 x, 4$ bougies; d'où une consommation spécifique de r,69 wall par bougie internationale ; celle-ci s'abaisse à $\mathrm{x}, 49$ watt si l'on ne tient pas compte des pertes dans le cuivre et le fer de la bobine de réactance, dans le solénoïde et son noyau, et dans le transformateur. Ce rendement, à peine attcint par les lampes à filament métallique, constitue l'une des plus belles conquêtes de la lumière Moore.

On peut toucher le tube sans éprouver de sensation de brûlure. D'ailleurs toute la chaleur est réparlie sur une surface de 5 , I 8 mètres carrés.

Mais la supériorité réelle du sysstème Moore réside dans la qualité et l'uniformité de son éclairage provenant de ce que la lumière se trouve nalurellement diffusée sans aucun des artifices auxquels on est contraint de recourir pour d'autres lampes, tels que globes dépolis, écrans, réflecteurs, éclairage indirect, qui causent une perte de ro à 50 pour too de la lumière initiale. Son ton chaud, légèrement rosé dans te cas de l'azote, plaît à l'ocil, el convient aussi bien pour les éclairages intérieurs qu'extéricurs. L'anhydride carbonique, qui donne une lumière blanche, abaisse la consonmation spécifique à 0,73 walt pour unc bongie et une tranche de I $\mathrm{cm}$ de largeur. On pourrait sans doute uliliser to néon, préparé suivant les procédés de Georges Claude, ol l'emploi de ce gaz réduirait la consommation spécifique probablement à moins de $\mathrm{I}$ wall.

Certaines usines génératrices pourraient sans doule soulever quelques difficultés à l'admission des tubes Moore sur leurs réseaux à cause de leur grand déphasage dans le circuit primaire ; mais la plupart d'entre elles cependant tolèrent déjà des récepteurs ayant un factour de puissance de 0,6 ; il n'y a donc pas d'objections scricuses à prévoir de ce côtó.

Au point de vue de l'applieation, on perd avee la lumiere Monre tous les avantages du montage en parallice qui permet de répartir l'énergie électrique entre plusieurs récepteurs ; car Moore a démontré hui-même que le rendement est d'autant meilleur que l'installation cist plus grande, c'est-à-dire le lube plus long, car les walls dissipés dans le cuive el te fer ne croissont pas proportionnollement à l'importance de l'installation. P'our le noment done, on limilera son emploi aux espaces de grande étendue aver $u n$ pelit íclarage de secours.

J.

\section{NOTE SUR LE TRAVAIL ET LA FLÈCHE DES LIGNeS AÉRIENNES EN CUIVRE}

Nous ayons rémi ici quelques domnées pratiqurs pour te calcul des lignes aŕriennes an point de voe mócanique(").

Ce calcul, effectué en tenant comple des dernieres prescriplions de l'Associalion des Ingćnieur's blectricirns allemands, par une méthode graphique lrès simple, nous a

(*) Bulletin de l'Instilut Electrotechnique de Grenoble. 
donné, pour les tensions, tes portées el les tempéralures ordinaires des résultats intéressants pour les industriels et les bureaux d'études ; ces résultats, consignés dans les tables qui se trouvent à la fin de cet article, permetlent de trouver très rapidement, pour une température et une portée donnces, la llèche et la tension à admettre dans chaque cas.

II est entendu qu'on ne pourra employer du cuivre mou, recuit, que lorsque la tension de travail ne dépassera pas $5 \mathrm{~kg}$. par millimètre carré. Pour les fils de cuivre étiré, la tension maxima admise est de $\mathrm{r}_{2} \mathrm{kgs}$, à moins que les limites d'élasticité et de ruplure n'aient été reconnues plus éloignées, par des essais préalables. Dans de tels cas, on peut admeltre une tension égale à la moitié de la limile d'élaslicité. Grâce à cette tolérance, il a été possible de porter la tension dans certains cas jusqu'à $16 \mathrm{kgs}$ par millimètre carré.

Pour le calcul des lignes, les prescriptions de l'Association des Ingénieurs électriciens allemands indiquent que le calcul sera fait une première fois en se basant sur une température de - $20^{\circ}$, et sans aucune surcharge, et une seconde fois en se basant sur une tempéralure de $-5^{\circ}$, en admetlant une surcharge produite par la glace el calculée par la formule empirique suivante :

$$
P=0,0 \times 5 q
$$

$P$ étant le poids de la glace en $\mathrm{kg}$ par mèlre courant et $q$ la section du fil en millimètre carrés.

Les considérations qui ont conduil à l'adoption de celte formule sont indiquées dans les paragraphes suivants :

"La détermination des surcharg̣es dues à la glace donne lieu à de grandes difficultés, car, en effet, il faul tenir compte de ce que la densité de la glace varie de 0,1 à $x$. On a remarqué que la couche de glace est plus forte pour les grandes sections que pour les petiles, et, par suite, on a été naturellement conduit à admettre la proportionnalité entre les sections el la surcharge correspondante.

"De nombreux exemples ont prouvé que la formule $P=$ $0,0 r 5 q$ indique la charge maxima de glace d'une façon très approchée. La Commission d'études remarqua que la surcharge maxima due à la glace et la surcharge maxima due au vent se produisent très rarement simullanément, car on a observé (du moins en Allemagne) que les vents les plus forts sont généralement des vents chauds. Comme l'ont montré de nombreux calculs, la surcharge maxima due à la glace est tonjours supérieure à celle produite par le vent, même pour de petites sections, il cst donc inulile de tenir compte du vent, puisqu'en calculant la ligne par la considédération de la charge de glace, elle esı par cela même largement calculée pour le vent. "

Dans la troisième partie des prescriptions, il est dit que, pour les traversées de routes, chemins de fer, elc..., on peul se dispenser d'un filet protecleur si la tonsion admise est inférieure à la moitié de la charge maxima admissible.

r'Administration allemande des posles a prescril, dans une circulaire en date du 8 fóvrier rgos, que, pour de tels croisements, on ne devait employer que du cuivre étiré ayant une lension de rupture d'at moins ho $\mathrm{kg}$ par $\mathrm{mm}^{2}$, et une seclion maxima d'au moins $35 \mathrm{~mm}^{2}$; le coefficient de sécurité devant ctre do //10, la charge maxima ne deva done pas dépasser $4 \mathrm{~kg}$ par millimelre carré.

Pour déterminer le travail mécanique et la fleche du fil dans les différents cas, en tenant comple de linfluence de la température, du poids de la ligne et des surcharges dues au vent et à la gace, on peut se servir des tables suivantes qui ont été établies d'après une mélhode exposće par $\mathrm{G}$. Nicolacus dans l'Electrolechnische Zeitschrift de rgo $_{7}$.

La méthode de Nicolaeus repose sur les deux égalités fondamentales suivantes :

$$
\begin{gathered}
t=\frac{\delta l^{2}}{\delta p} \\
t=\frac{\delta^{2} l^{2}}{24 p^{2} 0}-\frac{p \alpha}{\theta}
\end{gathered}
$$

dans lesquelles : $f$ est la flèche en centimètres ; o la densité du métal employé exprimée en $\mathrm{kg}$ par $\mathrm{cm}^{2}$ (c'est-à-dire, dans le cas du cuivre, $\left.\delta=0,008_{9}\right) ; l$ la portée en centimètres ; $p$ la tension du fll $\epsilon n \mathrm{~kg}$ par $\mathrm{cm}^{2} ; t$ la lempérature en degrés centigrades ; $\theta$ le coefficient de dilatalion linéaire (pour le cuivre $0=0,0000 \mathbf{r}_{7}$ ) $\alpha=\mathrm{r}: E, E$ étant le coefficient d'élasticité du métal employé (pour le cuivre, $E=$ $\mathrm{r} .300 .000 \mathrm{~kg}$ par $\mathrm{cm}^{2}$ ).

En remplaçant les lettres par leur valeur numérique,on a :

$$
\begin{gathered}
f=0,0 \circ \mathrm{I} \frac{l^{2}}{p} \\
t=0,194 \frac{p^{2}}{l^{2}}-0,453 p
\end{gathered}
$$

Ces égalités ne liennent pas comple des surcharges dues à la glace el au vent.

A l'aide de ces deux égalités, on peut construire deux familles de courbes ayant en abcisse les portées $l$ et en ordonnée les températures $t$.

La première famille donnera $p$ en fonction de $l$ et de $t$, tandis que la seconde famille donnera $f$ en fonction de $i$ et $t$.

Ces deux familles de courbes se couperont et chaque point d'intersection donnera 4 valeurs correspondantes de $p l f t$.

Nous donnons, dans les lables I à III ci-jointes, les différentes valeurs calculées par celte méthode. Ces résultats, comme d'ailleurs tous ceux obtenus par des procédés graphiques, sont empreints d'une légère inexaclitude, car certains points doivent être obtenus par interpolation.

Toutefois, les chiffres donnés dans les tables suivantes sont d'une exaclitude suffisante pour la pratiqui.

Les tables indiquent, pour une portée et une température données, la flèche et la tension correspondantes, calculées de façon que, pour une température de $-5^{\circ}$, et une charge de glace de $0,015 \mathrm{~kg}$ par $\mathrm{cm}^{2}$ (qui, comme nous l'avons dit plus haut, correspond à une surcharge maxima) la tension maxima admissible ne soit pas dépassée.

La table I se rapporte au fil de cuivre étiré normal, pour lequel on admet une tension maxima de $\mathrm{r}_{2} \mathrm{~kg}$ par centimètre carré.

Ia table II se rapporte au fil de cuivre étiré, particulièrement dur, dont la tension maxima peut être portée à I $6 \mathrm{kgs}$.

La table III se rapporle au fil de cuivre normal, quand la tension maxima ne doil pas dépasser 3 à $4 \mathrm{kgs}$ par $\mathrm{mm}^{2}$, c'est-ì-dire au cas où on supprime ics filets prolecteurs pour les traversées de route, chemins de fer, etc.

Les lables de I ì III ne sont valables que si les deux points d'altache d'une même portéc sont dans le même plan horizontal. Si ce n'est pas le cas, on peut encore se servir des tables en y apportant la modificalion suivante :

L'égalité (r) qui donne $f$ en fonction de $l$ reprósente une parabole.

Soient $A$ el $\mathrm{C}$ les points d'attache (voir la figure). La courbe que fail le fil en $\triangle B C$ peul ètre considérée comme une por- 
tion de la parabole DABC, D étant dans l'intersection de la parabole el du plan horizontal passant par $\mathrm{C}$.

Si on connaît le paramètre $F$ de la parabole DABC, et si on se donne la portée AC el l'angle $\alpha$, la corde DC est donnée par la formule

$$
\mathrm{DC}=l \cos \alpha+2 F \text { tang. } \alpha
$$

On peut calculer le paramètre des paraboles correspondant aux portées et aux flèches indiquées dans les tables à l'aide de la formule :

$$
F=\frac{l^{2}}{8 f}
$$

Si on part de la condition que à - $-5^{\circ}$, el avec surcharge de glace, la tension du fil ne doil pas dépasser ha valeur maxima admise, $p$, il faul porter la corde AC dans la parabole yui correspond au $p^{\prime}$ donné pour - $5^{\circ}$ et surcharge de glace. Le paramètre de celle parabole est :

$$
\begin{array}{rlrl}
\text { Pour } p^{\prime} & =12 \mathrm{kgs} \text { par } \mathrm{m} / \mathrm{m}^{2}: F & =500 \mathrm{~m} . \\
n & =16 ", \quad & F & =670 \mathrm{~m} .
\end{array}
$$

lirmarquons, en passant, que la llèche correspondant à $p^{\text {' }}$ domne d'ailleurs une valeur approchée de la plus grande fleche possible. Les tables montrent en effet que la flèche correspondant à - $5^{\circ}$ et surcharge de glace est la plus crande, ou tout au moins s'approche de la plus grande de liès près.

Comnaissanl $l$ et $\alpha$, on calcule DG. On peul dès lors se scrvir des tables qui domnent la flèche el la tension aux diverses températures; les variations de température et de charge influeront alors sur la tension du fil en C comme si ABC faisail partic de la parabole DABG. En réalité, la con-

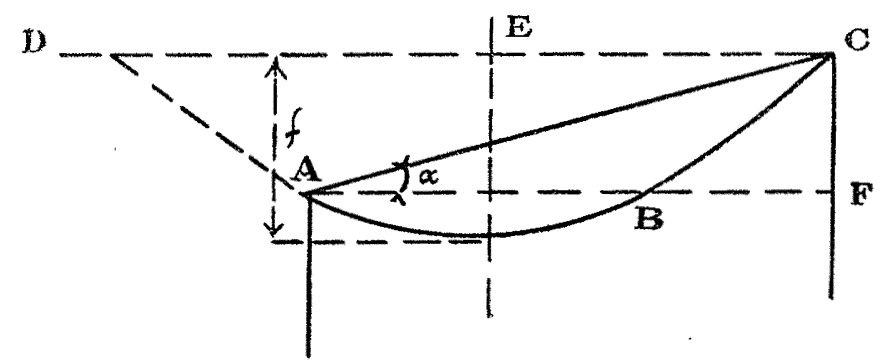

formation de la partie $\mathrm{ABC}$ varie avec la température, et les paraboles complétées correspondantes ne donnent pas toujours la même valeur pour DC. Cependant, si on ne considère que la tension du fil, ces divergences peuvent être négligées, car on remarquera dans les lables que, pour une lempérature déterminée, la tension varie beaucoup plus lentement que la portée.

L'approximation obtenue de celle manière est d'autant plus grande que l'angle $\%$ est plus petit, et que, pour une porlée donnée $D C$, les paramètres des paraboles correspondant aux différentes températures diffèrent moins les uns des aulres, c'est-à-dire que les portées deviennent plus grandes. Si on pouvait complètement négliger les variations le paramètre, ce qui est le cas pour de très grandes portées, les paraboles seraient les mêmes pour toutes les températures, c'est-à-dire que l'arc de parabole symétrique DAG ne diffèrerait de l'arc $A B G$ que parce que le point $A$, dans le premier cas, est soutenu par la partie DA, et dans le second cas, est supporté par un appui.

Le fait que la plupart des travaux des lignes s'effectuent en élé, avec des températures d'environ $10^{\circ}$, ou tout au moins supérieures à o, favorise l'emploi de cctte méthode ; car, plus la température est élevée, plus le paramètre se rapproche de celui de la parabole correspondant à $p$ maximim.
Pour terminer, nous donnons un exemple de l'emploi des tables dans le cas de supports à des niveaux différents :

$$
\text { Soit : } \quad \mathrm{AC}=l=60 \mathrm{~m} . \quad \lg \alpha=0,04
$$

c'est-à-dire qu'un des supporls se trouve à $2^{\text {m }} 40$ au-dessus de l'autre. Admeltons de plus une tension maxima de $12 \mathrm{kgs}$ par $\mathrm{mm}^{2}$.

D'après l'égalité (3), on a en posant $\cos \alpha=\mathrm{I}$ :

$$
\mathrm{DC}=60+2 \times 500 \times 0,04=100 \mathrm{~m} .
$$

Le fil doit done être tendu d'après les valeurs domnces dams la table 1, pour une portée de 100 mètres.

\begin{tabular}{|c|c|c|c|c|c|c|c|c|c|c|c|c|c|c|}
\hline \multirow{2}{*}{$\begin{array}{c}t \\
{ }^{\circ} \mathrm{C} .\end{array}$} & \multicolumn{2}{|c|}{$l=40 \mathrm{~m}}$. & \multicolumn{2}{|c|}{$l=60 \mathrm{~m}}$. & \multicolumn{2}{|c|}{$l=80 \mathrm{~m}$} & \multicolumn{2}{|c|}{$l=100 \mathrm{~m}}$. & \multicolumn{2}{|c|}{$l=120 \mathrm{~m}}$. & \multicolumn{2}{|c|}{$l=140 \mathrm{~m}$} & \multicolumn{2}{|c|}{$l=160 \mathrm{~m}$} \\
\hline & $p$ & i & $p$ & $f$ & $p$ & i & $p$ & $r$ & $p$ & f & $p$ & i & $p$ & $f$ \\
\hline \multicolumn{15}{|c|}{ POIDS PROPRE SEUL } \\
\hline-20 & 12,1 & 14 & 9,5 & 但 & 7,1 & 100 & 5,7 & 185 & 5,1 & 1305 & 0,0 & 445 & 5,0 & 600 \\
\hline-10 & 10,3 & 17 & 8,0 & 50 & 6,2 & 113 & 5,3 & 205 & 4,95 & 320 & $\downarrow, 9$ & 460 & 4,9 & 610 \\
\hline & 8,6 & 20 & 6,9 & 59 & 5,5 & 126 & 4,9 & 220 & 4,8 & 335 & 4,8 & $475)$ & 4.8 & 620 \\
\hline+10 & 7,0 & 24 & 5,9 & 69 & 5,0 & 140 & 4,6 & 237 & 4,6 & 350 & 4,7 & 490 & 4,7 & 630 \\
\hline+20 & 5,6 & 28 & 5,0 & 81 & 4,5 & 155 & 4,3 & 254 & 4,4 & 366 & 4,5 & 505 & 4,6 & 640 \\
\hline+30 & 4,6 & 32 & 4,2 & 93 & 4,1 & 174 & 4,1 & 268 & 4,2 & 1382 & 4,3 & 525 & 4,5 & 1650 \\
\hline \multicolumn{15}{|c|}{ POIDS PROPRE ET SURCHARGE } \\
\hline-5 & 12,0 & 40 & 2,0 & 89 & g, & 150 & 12. & 2 & 12 & 1358 & 1100 & 150 & 108 & \\
\hline
\end{tabular}

Dans les tables que nous donnons ci-joint : $l$ représente la portćc en mètres ; $t$ la température en degrés $\mathrm{C}$. ; $p$ la

\begin{tabular}{|c|c|c|c|c|c|c|c|c|c|c|c|c|c|c|}
\hline \multirow{2}{*}{$\begin{array}{c}t \\
{ }^{\circ} \mathrm{C}\end{array}$} & \multicolumn{2}{|c|}{$1=80 \mathrm{~m}$} & \multicolumn{2}{|c|}{$l=00 \mathrm{~m}$} & \multicolumn{2}{|c|}{$l=120 \mathrm{~m}}$. & \multicolumn{2}{|c|}{$l=140 \mathrm{~m}}$. & \multicolumn{2}{|c|}{$l=16) \mathrm{m}}$. & \multicolumn{2}{|c|}{$l=8 . \mathrm{m}$} & \multicolumn{2}{|c|}{$l=200 \mathrm{~m}$} \\
\hline & \multirow[t]{2}{*}{$p$} & \multirow[t]{2}{*}{$f$} & \multirow{2}{*}{${ }^{p}$} & \multirow{2}{*}{ t. } & \multirow{2}{*}{$p$} & \multirow{2}{*}{-1} & \multirow{2}{*}{\multicolumn{2}{|c|}{$\overbrace{}^{p}$}} & \multirow{2}{*}{$p$} & \multirow{2}{*}{$f$} & \multirow{2}{*}{$p$} & \multirow{2}{*}{${ }^{f}$} & \multirow[t]{2}{*}{$p$} & \multirow[t]{2}{*}{ t } \\
\hline & & & & & & & & & & & & & & \\
\hline-20 & 13,1 & 55 & 11,0 & 10 & 9,2 & 165 & 8,1 & 265 & $7, \bar{i}$ & 375 & 7,1 & 490 & & 630 \\
\hline-10 & 11,5 & 65 & 0,8 & 115 & 8,4 & 185 & 7,6 & 285 & 7,2 & 395 & 6.85 & 515 & 6,8 & 650 \\
\hline & 16,0 & 75 & 8,8 & 130 & 7,7 & 205 & 7,1 & 305 & 6,9 & 415 & 6,6 & 535 & 6,6 , & 670 \\
\hline+10 & 8,8 & 85 & 7,9 & 145 & 7,1 & 225 & 6,7 & 320 & 6,6 & 430 & 6,4 & $55 \%$ & 6,5 & 690 \\
\hline+20 & 7,7 & 95 & 7,0 & 160 & 6,6 & 240 & 6,4 & 335 & 6,3 & 445 & 6,25 & 570 & 5,3 & 710 \\
\hline+30 & 6,8 & & 6,3 & 175 & 6,1 & 255 & 6,11 & 350 & 6,1 & 4.60 & 6,1 & 585 & & 730 \\
\hline & & & & & s & & E & & A AR & & & & & \\
\hline- & 160 & 119 & 16.8 & & 10 & & 1160 & & $\{16,0\}$ & & 16, & 106 & & +46 \\
\hline
\end{tabular}
tension spécilique en kg. par $\mathrm{mm}^{2}$; $f$ la flèche en centimètres.

TABLE I. $-p$ maximum $=\mathrm{r}_{2} \mathrm{kgs}$ par $\mathrm{mm}^{2}$.

TABLE II. $-p$ maximum $={ }_{1} 6 \mathrm{kgs}$ par $\mathrm{mm}^{2}$.

TABLE III. $-p$ maximum $=4 \mathrm{kgs}$ par $\mathrm{mm}^{2}$.

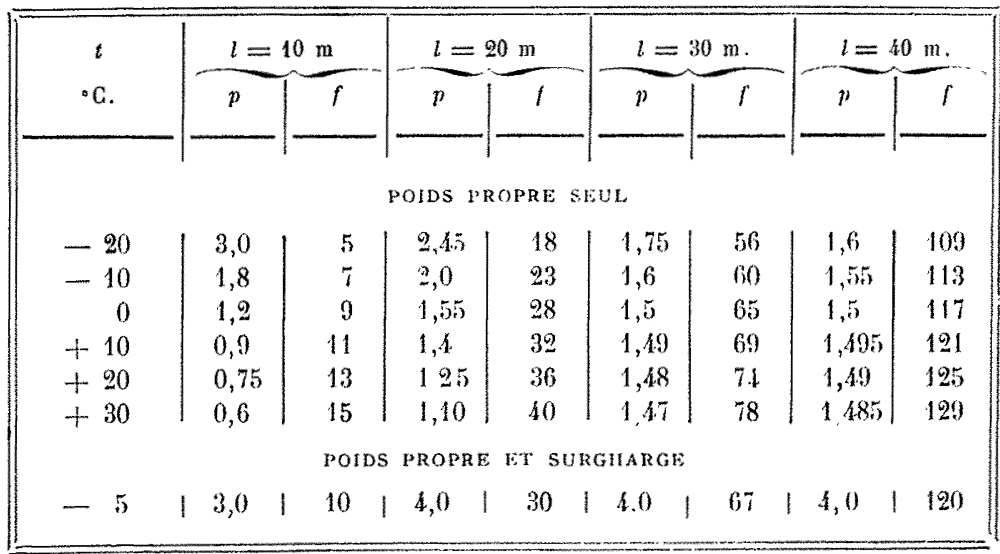

Au moment de la pose, le fil (poids spécilique 8,9 ) soumis à une charge due uniquement à son propre poids doit être 
tendu suivant la tempéralure, de façon à obtenir la tension et la flèche indiquées par les lables. Les valeurs des tensions et des flèches portées dans les tables sont choisies de telle sorte que, lorsque au poids du fil viennent s'ajouter des surcharges accidentelles (glace ou vent) de $0,015 \mathrm{~kg}$ par mètre et $\mathrm{mm}^{2}$, et pour une température de $-5^{\circ} \mathrm{c}$, le fil ne soit pats soumis à une tension supéricure à la tension maxima indiquée à la dernière lignne de chaque table.

Arribert el Baron,

makniturs-alectriciens $I$. E. G.

\section{ACADÉMIE DES SGIENGES}

\section{MÉCANIQUE ET ÉLECTRICITÉ}

Sur l'existence de deux potentiels explosifs, note de M.M. P. VILARD el H. Abrallam. Sćances des 23 mai et 25 juillet 1910 .

On sait que, lorsqu'on élève progressivement lo voltage aux bornes d'un éclateux à ćlcctroles sphériques, on voit à un certain moment des aigrelles apparaître, puis l'étincelle disruptive éclate 't decharge complètement les conducteurs, empechant par ccla mème la diffírence de potentiel de crồtre davantage ; on a atteint 1. polentiel explosif ordinaire que nous désignerons d'une manière générale par $V$ '.

Nous avons reconnu que, moyennant quelques précautions, ce vollage $F$, en apparence infranchissable peut être considérablement dépassé sans qu'il se produise aucune élincelle. Cet accroisement de la tension est cependant limité, et, pour un éclateur domné, il existe, outre le potentiel $V^{\prime}$, un deuxième potenticl explosi? pouvant elre le double du premier et caractérisé par un régime foul à fait différent de préparation à l'étincelle, l'éclatement de cette dernière étant précédé non plus par l'apparition des aigrettes, mais par la formation, sur l'anode, d'une lueur persistante et continue, visible même en plein jour.

Pour isoler ec phénomène el l'observer dans l'air ordinaire qui n'est pas cependant le gaz le plus favorable à l'expérience, il convient de constituer l'éclateur par une anode sphérique de petit diamètre ( $\mathrm{r} \mathrm{cm}$. environ) et une cathode de grande surface, telle qu'une grosse sphère ou un large plateau à bords arrondis. La source électrique sera de préférence une machine statique puissante, reliéc au besoin à des condensateurs destinés à régulariser le régime ; on aura soin d'éviter les contacts imparfaits dans les commexions.

Cela étant, et le voltage $V$ 'étant atteint, il arrive très fréquemment, à la faveur des inévitables "ratés " de l'étincelle de décharge, que ce potenliel puisse être neltement dépassé. Aussitôt les aigreltes positives, qu'on s'attendait à voir augmenter d'importance, disparaissent complètement ct définitivement pour faire place à un aspect tout à fait différent. L'anode, sur toute la zone en regard de la cathode, se recouvre d'une gaine luminescente, d'un rose violacé, qui augmente d'éclat et d'étendue à mesure que lit différence de potentiol s'élève ; le reste du champ est tout à fait obsenr ct aucune lueur ne se montre sur la cathode ; d'autre part, un vent électrique intense, sensible à une distance de plus de $\mathrm{x}$ m. s'échappe de la région qui avoisine l'anode $\left(^{*}\right)$.

Elevant encore le voltage, on voit la gaine lumincuse se renforcer à son sommet, puis donner naissance à une pointe plus brillante dirizée vers la calhode ; ce pointement est l'amorce de la nouvelle étincelle dismplive qui se produit tout aussitôt. Le second potentiel explosif f" est alors alteint.

(*) Plusieurs observations de Nicholson ( 1787 ), de Faraday et de diver autres physiciens, sur les décharges par heurs (glowdischarge, glimmstrom) et sur certains retards à la decharge, se rat achent tantôt au phenomène bien défini qut nous décrivons, tantôt à d'autres modes dé décharges qui en sont tout à fait distincts.
Si; avant d'atteindre colte nouvelle valeur, on rétrogradait vers $V$ ', l'étincelle ou tout au moins l'aigrette réapparaîtrait comme dans l'expórience faite par vollages eroissants $\left(^{\star}\right)$.

On peut aussi opérer à voltage constant et faire varier la dis. tance des électrodes. On trouve alors pour chaque polentiel deux distances explosives, tandis qu'on n'a jamais d'étincelle pour les distances intermédiaires.

Ainsi, pour le premier polentiel explosif, $I^{\prime}$, seul observé elt général, l'étincelle est sous la dépendance des irrégularités de l'aigrelle préparatoire el constituc, en quelque sorte, un accident qu'il est possible d'éviter. Le second potentiel $V^{\prime \prime}$, au contraire, scmble correspondre à un phénomène normal à préparation régulière. Entre ces deux valeurs, le régime luminescent est parfaitemenl stable et une étincelle n'est possible que par l'intervention d'une action étrangère.

La composition complexe de l'air atmosphérique semble avor une influence considérable sur l'ćtablissement du régime de la gaine anodique et sur l'éclatement accidentel de l'étincelle de bas voltage. On réussit mieux l'expérience avec de l'air pur et sec yu'on renouvelle dès qu'on y fait éclater quelques étincelles. Darts de l'azote sec le phénomène devient plus brillant et vraiment remarquable. Il n'est plus nécessaire d'avoir une anode de pelif diamètre, ef l'on peul même constituer l'exploscur par des boules étales de 4 ou $5 \mathrm{~cm}$. de dirmètre. Dans ce gaz, le passage par la valeur $\gamma$ ' est toujours exactement indiqué par l'apparition de la gaine anodique, el cependant on n'aperçoit que de faibles aigrettes, qui peuvent mème ne pas se produire dans une expérience conduite doucement. Le caractère accidentel de la première étincelle devient alor's toul à fail manifeste $\left(^{\star \star}\right)$.

Les phénomènes que nous venons de décrire peuvent ètre observé: sous différentes pressions, et on les suit aisément jusque vers $2 \mathrm{~mm}$. de mercure.

L'expérience réussit également très bien dans l'hydrogène.

L'établissement de la luminescence anodique est rendu au contraire incertain puis impossible par la présence de traces de gaz carbonique ou sulfureux on de quantités notables de vapeur d'eau. C'est ainsi que si l'on a établi ce régime dans l'air ordinaire, il suffit de souffler avec la bouche sur l'anode pour faire éclater l'étincelle.

La luminescence de l'anode, caractéristique des potentiels com. pris entre $V^{\prime}$ et $V$ ', est l'indice évident du passage d'importantes quantités d'électricité. On constate en effet que, pour maintenir ce régime, mêrne à des voltages modérés, on est obligé de recourir à l'emploi d'une machine statique à platcaux mulliples. Nous nous sommes servis d'une machine Bonelli-Roycourt sans secteurs à six plateaux d'ébonite.

Dans ces conditions, l'intensité du courant absorbé par l'éclateur augmente avec le diamètre de l'anode el peut, sous une cenlaine de mille volts, être de l'ordre du milliampère. La puissance ainsi localement dépenséc sur l'anode, tout aussi importante quo celle exigée par d'autres modes d'illumination électrique des gaz, montre qu'il s'agit non d'un courant ordinaire d'ionisation, mais d'une décharge véritable. Au surplus, la densité du courant sur l'anode est tout à fait comparable à celle qu'on admet dans l'ćlectrolyse d'un liquide.

Voici, à ce sujel, les résultats des deux expériences failes à la pression atmosphérique dans de l'azole sec (azote industricl, procédés G Claude), avec des anodes sphériques placées en regard d'une cathode plaue à bords arrondis :

(*) Le phénomène devient tout à fait frappant en chargeant, à un voltage compris entre $V^{\prime}$ et $V^{\prime \prime}$, un condensateur relié à l'éclateur. Aucune étincelle ne jaillira aussi longtemps que le fonctionnement de la source électrique maintiendra cette tension. Mais si l'on arrete la machine, le potentiel baisse spontanément peu à $p^{-} u$, et, au moment où il repasse par $V$, des aigrettes se produisent; suivies aussitôt par l'étincelle disruptive qui décharge complètement le condensateur.

(**) Rappelons à ce sujet que M. J. J. Thomson a observé des élévations considérables da potentiel explosif dans les gaz rigourcusement secs. 